\title{
A Note on the Perturbation of MF Algebras and Quasidiagonal C*-Algebras
}

\author{
Wenjuan Zhan, Liguang Wang \\ School of Mathematical Sciences, Qufu Normal University, Qufu, China \\ Email: zhanwenjuan0624@163.com, wangliguang0510@163.com
}

How to cite this paper: Zhan, W.J. and Wang, L.G. (2019) A Note on the Perturbation of MF Algebras and Quasidiagonal $\mathrm{C}^{*}$-Algebras. Journal of Applied Mathematics and Physics, 7, 2026-2030. https://doi.org/10.4236/jamp.2019.79139

Received: August 5, 2019

Accepted: September 17, 2019

Published: September 20, 2019

Copyright $\odot 2019$ by author(s) and Scientific Research Publishing Inc. This work is licensed under the Creative Commons Attribution International License (CC BY 4.0).

http://creativecommons.org/licenses/by/4.0/

(c) (i) Open Access

\begin{abstract}
Perturbation problem of operator algebras was first introduced by Kadison and Kastler. In this short note, we consider the uniform perturbation of two classes of operator algebras, i.e., MF algebras and quasidiagonal $\mathrm{C}^{*}$-algebras. We show that the sets of MF algebras and quasidiagonal $\mathrm{C}^{*}$-algebras of a given $C^{*}$-algebra are closed under the perturbation of uniform norm.
\end{abstract}

\section{Keywords}

MF Algebra, Quasidiagonal C*-Algebra

\section{Introduction and Preliminaries}

Kadison and Kastler in [1] initiated the study of uniform perturbations of operator algebras. They considered a fixed $\mathrm{C}^{*}$-algebra $\mathscr{U}$ and equipped the set of all $\mathrm{C}^{*}$-subalgebras of $\mathscr{U}$ with a metric arising from Hausdorff distance between the unit balls of these subalgebras. We first recall the following definition of the metric $d$ defined on the set of all $\mathrm{C}^{*}$-subalgebras of a $\mathrm{C}^{*}$-algebra $\mathscr{U}$ (see [1]).

Definition 1.1. Let $\mathscr{A}$ and $\mathscr{B}$ be $\mathrm{C}^{*}$-subalgebras of a $\mathrm{C}^{*}$-algebra $\mathscr{U}$. The Kadison-Kastler metric $d(\mathscr{A}, \mathscr{B})$ between $\mathscr{A}$ and $\mathscr{B}$ is defined by

$$
d(\mathscr{C}, \mathscr{B})=\max \left\{\sup _{a \in(\mathscr{Y})_{1}} \inf _{b \in(\mathscr{O})_{1}}\|a-b\|, \sup _{b \in(\mathscr{O})_{1}} \inf _{a \in(\because)_{1}}\|a-b\|\right\}
$$

where $(\mathscr{C})_{1}$ and $(\mathscr{B})_{1}$ denote the unit ball of $\mathscr{A}$ and $\mathscr{B}$ respectively.

Kadison and Kastler conjectured in [1] that sufficiently close von Neumann algebras (or $\mathrm{C}^{*}$-algebras) are necessarily unitarily conjugate. The first positive answer to Kadison-Kastler's conjecture was given by Christensen [2] when either

or $\mathscr{B}$ is a von Neumann algebra of type I. Many results related to this conjecture have been obtained during the past 40 years ([3] [4] [5] [6]). One-sided 
version of Kadison-Kastler's conjecture was introduced and studied by Christensen in [4] as well. Christensen showed in [4] that a nuclear $\mathrm{C}^{*}$-algebra that is nearly contained in an injective von Neumann algebra is unitarily conjugate to this von Neumann algebra. Christensen, Sinclair, Smith and White showed in [5] that the property of having a positive answer to Kadison's similarity problem transfers to close $C^{*}$-algebras. Very recently, Kadison-Kastler's conjecture has been proved for the class of separable nuclear $\mathrm{C}^{*}$-algebras in the remarkable paper [6].

The problem we are going to consider is as follows: Suppose $\mathscr{A}, \mathscr{B}$ are $\mathrm{C}^{*}$-subalgebras of a $\mathrm{C}^{*}$-algebra $\mathscr{C}$. If $d(\mathscr{C}, \mathscr{H})<\gamma$, is $\mathscr{A}$ and $\mathscr{B}$ share similar properties?

In this short note, we show that the sets of matricial field algebras (MF algebras) and quasidiagonal $C^{*}$-algebras of a given $C^{*}$-algebra are closed under the perturbation of uniform norm.

\section{Main Results}

In this section, we consider some topological properties of the set of all MF algebras and quasidiagonal $\mathrm{C}^{*}$-subalgebras under the perturbation of uniform norm. For basics of $\mathrm{C}^{*}$-algebras, we refer to [7] and [8]. We first recall the definition of MF algebras ([9]).

Suppose $\left\{\mathcal{M}_{k_{n}}(\mathbb{C})\right\}_{n=1}^{\infty}$ is a sequence of complex matrix algebras. We can introduce the full $\mathrm{C}^{*}$-direct product $\prod_{m=1}^{\infty} \mathcal{M}_{k_{m}}(\mathbb{C})$ of $\left\{\mathcal{M}_{k_{n}}(\mathbb{C})\right\}_{n=1}^{\infty}$ as follows:

$$
\prod_{n=1}^{\infty} \mathcal{M}_{k_{n}}(\mathbb{C})=\left\{\left(Y_{n}\right)_{n=1}^{\infty} \mid \forall n \geq 1, Y_{n} \in \mathcal{M}_{k_{n}}(C) \text { and } \sup _{n \geq 1}\left\|Y_{n}\right\|<\infty\right\} \text {. }
$$

Furthermore, we can introduce a norm closed two sided ideal in $\prod_{n=1}^{\infty} \mathcal{M}_{k_{n}}(\mathbb{C})$ as follows,

$$
\sum_{n=1}^{\infty} \mathcal{M}_{k_{n}}(\mathbb{C})=\left\{\left(Y_{n}\right)_{n=1}^{\infty} \in \prod_{n=1}^{\infty} \mathcal{M}_{k_{n}}(\mathbb{C}): \lim _{n \rightarrow \infty}\left\|Y_{n}\right\|=0\right\}
$$

Let $\pi$ be the quotient map from $\prod_{n=1}^{\infty} \mathcal{M}_{k_{n}}(\mathbb{C})$ to $\prod_{n=1}^{\infty} \mathcal{M}_{k_{n}}(\mathbb{C}) / \sum_{n=1}^{\infty} \mathcal{M}_{k_{n}}(\mathbb{C})$. It is known that $\prod_{n=1}^{\infty} \mathcal{M}_{k_{n}}(\mathbb{C}) / \sum_{n=1}^{\infty} \mathcal{M}_{k_{n}}(\mathbb{C})$ is a unital $C^{\star}$-algebra. If we denote $\pi\left(\left(Y_{n}\right)_{n=1}^{\infty}\right)$ by $\left[\left(Y_{n}\right)_{n}\right]$, then

$$
\left\|\left[\left(Y_{n}\right)_{n}\right]\right\|=\limsup _{n \rightarrow 1}\left\|Y_{n}\right\| \text {. }
$$

Now we are ready to recall an equivalent definition of MF algebras which is given by Blackadar and Kirchberg ([9]).

Definition 2.1. (Theorem 3.2.2, [9]) Let $\mathscr{U}$ be a separable $\mathrm{C}^{*}$-algebra. If

can be embedded as a $\mathrm{C}^{*}$-subalgebra of $\prod_{n=1}^{\infty} \mathcal{M}_{k_{n}}(\mathbb{C}) / \sum_{n=1}^{\infty} \mathcal{M}_{k_{n}}(\mathbb{C})$ for a sequence $\left\{k_{n}\right\}_{n=1}$ of integers, then $\mathscr{U}$ is called an MF algebra.

Lemma 2.2. ([10] Lemma 2.12) Suppose that $\mathscr{U}$ is a separable $C^{*}$-algebra. 
Assume for every finite family of elements $x_{1}, x_{2}, \cdots, x_{n}$ in $\mathscr{Z}$ and every $\varepsilon>0$, there is an MF algebra $\mathscr{T}_{1}$ such that $\left\{x_{1}, x_{2}, \cdots, x_{n}\right\} \subset_{\varepsilon} \mathscr{U}_{1}$, (in the sense of Definition 2.3 in [10]). Then $\mathscr{U}$ is also an MF algebra.

Proposition 2.3. Let $\mathscr{Z}$ be a $\mathrm{C}^{*}$-algebra and $\mathfrak{F}$ be the subset of all separable MF algebras contained in $\mathscr{U}$. Then $\mathfrak{F}$ is closed under the metric $d$.

Proof. Let $\mathscr{\mathscr { C }} \in \overline{\mathfrak{F}}$. Then there exist $\mathscr{I}_{n} \in F$ such that $d\left(\mathscr{C}_{n}, \mathscr{C}\right) \rightarrow 0$. For any $x_{1}, x_{2}, \cdots, x_{m} \in \mathscr{C}, \forall \varepsilon>0$, there is an $n_{0}$ such that

$$
\begin{gathered}
d\left(\mathscr{I}_{n_{0}}, \mathscr{J}\right)<\frac{\varepsilon}{2 \sum_{i=1}^{m}\left\|x_{i}\right\|+1} \text {. Then there exist } y_{1}, y_{2}, \cdots, y_{m} \in \mathscr{\mathscr { C } _ { n _ { 0 } }} \text { such that } \\
\qquad\left\|x_{i}-y_{i}\right\|<\frac{\varepsilon}{2 \sum_{i=1}^{m}\left\|x_{i}\right\|+1}\left\|x_{i}\right\|<\varepsilon
\end{gathered}
$$

for all $i$. It follows from Lemma 2.2 that $/$ is also a MF algebra.

We will recall some results about quasidiagonal $\mathrm{C}^{*}$-algebras for the reader's convenience. We refer the reader to [11] for a comprehensive treatment of this important class of $\mathrm{C}^{*}$-algebras.

Definition 2.4. A subset $\Omega \subset \mathcal{B}(\mathscr{H})$ is called a quasidiagonal set of operators if for each finite set $\omega \subset \Omega$, finite set $\chi \subset \mathscr{H}$ and $\varepsilon>0$, there exists a finite rank projection $P \in \mathcal{B}(\mathscr{K})$ such that $\|T P-P T\| \leq \varepsilon$ and $\|P(x)-x\| \leq \varepsilon$ for all $T \in \omega$ and $x \in \chi$.

Definition 2.5. A $C^{*}$-algebra $\mathscr{U}$ is called quasidiagonal (QD) if there exists a faithful representation $\pi: \mathscr{U} \rightarrow B(\mathscr{H})$ such that $\pi(\mathscr{U})$ is a quasidiagonal set of operators.

The following result is Lemma 7.1.3 in [11] which is useful to determine whether a $\mathrm{C}^{*}$-algebra is quasidiagonal or not.

Lemma 2.6. $\mathrm{A} \mathrm{C}^{*}$-algebra $\mathscr{U}$ is quasidiagonal if and only if for each finite set $F \subset \mathscr{U}$ and $\varepsilon>0$, there exists a completely positive map $\phi: \mathscr{\ell} \rightarrow M_{n}(\mathbb{C})$ such that

$$
\|\phi(a b)-\phi(a) \phi(b)\|<\epsilon
$$

and

$$
\|\phi(a)\|>\|a\|-\epsilon
$$

for all $a, b \in F$.

Proposition 2.7. Let $\mathscr{U}$ be a separable $\mathrm{C}^{*}$-algebra. Let $\mathfrak{F}=Q D(\mathscr{U})$ be the set of all quasidiagonal $C^{*}$-subalgebras of $\mathscr{U}$. Then $\mathfrak{F}$ is closed under the metric $d$.

Proof. Let $\mathscr{\mathscr { C }} \in \overline{\mathfrak{F}}$ and choose $\mathscr{I}_{n} \in \mathfrak{F}$ such that $d\left(\mathscr{C}_{n}, \mathscr{C}\right) \rightarrow 0$. Given finite subset $\left\{x_{1}, x_{2}, \cdots, x_{k}\right\}$ of the unit ball of $\mathscr{C}$ and $\epsilon>0$. There is a $N \in \mathbb{N}$ such that $d\left(/_{N}, \mathscr{C}\right)<\frac{\epsilon}{6}$. Choose $y_{1}, y_{2}, \cdots y_{k}$ in the unit ball of $\mathscr{C}_{N}$ such that $\left\|x_{i}-y_{i}\right\|<\frac{\epsilon}{6}$ for $i=1,2, \cdots, k$. Since $\mathscr{C}_{N}$ is $\mathrm{QD}$, it follows from Lemma 2.6 that there is a c.c.p. map $\phi: \mathscr{C}_{N} \rightarrow M_{t}(\mathbb{C})$ such that 


$$
\left\|\phi\left(y_{i} y_{j}\right)-\phi\left(y_{i}\right) \phi\left(y_{j}\right)\right\| \leq \frac{\varepsilon}{6}
$$

and

$$
\left\|\phi\left(y_{j}\right)\right\| \geq\left\|y_{j}\right\|-\frac{\epsilon}{6}
$$

for all $i, j=1,2, \cdots, k$. Now use Arveson's extension theorem ([11]) to extend $\phi$ to a c.c.p. map $\tilde{\phi}$ from $\mathscr{Q}$ to $M_{t}(\mathbb{C})$. Let $\psi: \mathscr{C} \rightarrow \mathbb{l}_{t}(\mathbb{C})$ be the restriction of $\tilde{\phi}$ to . Then for each $i, j=1,2, \cdots, k$, we have

$$
\begin{aligned}
& \left\|\psi\left(x_{i} x_{j}\right)-\psi\left(x_{i}\right) \psi\left(x_{j}\right)\right\| \\
& \leq\left\|\psi\left(x_{i} x_{j}\right)-\psi\left(x_{i} y_{j}\right)\right\|+\left\|\psi\left(x_{i} y_{j}\right)-\psi\left(y_{i} y_{j}\right)\right\| \\
& \quad+\left\|\psi\left(y_{i} y_{j}\right)-\psi\left(y_{i}\right) \psi\left(y_{j}\right)\right\|+\left\|\psi\left(y_{i}\right) \psi\left(y_{j}\right)-\psi\left(x_{i}\right) \psi\left(y_{j}\right)\right\| \\
& \quad+\left\|\psi\left(x_{i}\right) \psi\left(y_{j}\right)-\psi\left(x_{i}\right) \psi\left(x_{j}\right)\right\| \\
& <\varepsilon
\end{aligned}
$$

and

$$
\left\|\psi\left(x_{i}\right)\right\|=\left\|\psi\left(x_{i}-y_{i}\right)+\psi\left(y_{i}\right)\right\| \geq\left\|\psi\left(y_{i}\right)\right\|-\frac{\varepsilon}{6}>\left\|y_{i}\right\|-\frac{\varepsilon}{3} \geq\left\|x_{i}\right\|-\varepsilon .
$$

Use Lemma 2.6 again we have that $/ /$ is quasidiagonal.

\section{Conclusion}

In this paper, we use some characterizations of MF algebras and quasidiagonal $\mathrm{C}^{*}$-algebras to show that these two sets of $\mathrm{C}^{*}$-subalgebras of a given $\mathrm{C}^{*}$-algebras are closed with respect to the topology induced by the Kadison-Kastler metric.

\section{Founding}

Partially supported by NSFC (11871303 and 11671133) and NSF of Shandong Province (ZR2019MA039).

\section{Conflicts of Interest}

The authors declare no conflicts of interest regarding the publication of this paper.

\section{References}

[1] Kadison, R.V. and Kastler, D. (1972) Perturbations of von Neumann Algebras. I. Stability of Type. American Journal of Mathematics, 94, 38-54. https://doi.org/10.2307/2373592

[2] Christensen, E. (1974) Perturbations of Type I von Neumann Algebras. Journal of the London Mathematical Society, 9, 395-405.

https://doi.org/10.1112/jlms/s2-9.3.395

[3] Christensen, E. (1977) Perturbation of Operator Algebras. Inventiones Mathematicae, 43, 1-13. https://doi.org/10.1007/BF01390201

[4] Christensen, E. (1980) Near Inclusions of C*-Algebras. Acta Mathematica, 144, 249-265. 
https://doi.org/10.1007/BF02392125

[5] Christensen, E., Sinclair, A.M., Smith, R.R. and White, S.A. (2010) Perturbations of $\mathrm{C}^{*}$-Algebraic Invariants. Geometric and Functional Analysis, 20, 368-397. https://doi.org/10.1007/s00039-010-0070-y

[6] Christensen, E., Sinclair, A.M., Smith, R.R., White, S.A. and Winter, W. (2012) Perturbations of Nuclear C-Algebras. Acta Mathematica, 208, 93-150. https://doi.org/10.1007/s11511-012-0075-5

[7] Kadison, R.V. and Ringrose, J. (1983 and 1986) Fundamentals of the Theory of Operator Algebras, Vol. I and II. Academic Press, Orlando.

[8] Takesaki, M. (1979) Theory of Operator Algebras I. Springer Verlag, New York. https://doi.org/10.1007/978-1-4612-6188-9

[9] Blackadar, B. and Kirchberg, E. (1997) Generalized Inductive Limits of Finite Dimensional C-Algebras. Mathematische Annalen, 307, 343-380. https://doi.org/10.1007/s002080050039

[10] Hadwin, D., Li, J.K., Shen, J.H. and Wang, L.G. (2012) Reduced Free Products of Unital AH Algebras and Blackadar and Kirchberg's MF Algebras. Journal of Operator Theory, 69, 275-302.

[11] Brown, N.P. and Ozawa, N. (2008) C-Algebras and Finite-Dimensional Approximations, Graduate Studies in Mathematics. Vol. 88, American Mathematical Society, Providence. https://doi.org/10.1090/gsm/088 